\title{
Ética periodística para una información con perspectiva de género
}

Journalistic Ethics for Information with a Gender Perspective

Rubén Rivas-de-Roca

Doctorando en Comunicación

Investigador y profesor PIF de la Universidad de Sevilla

rrivasderoca@us.es
Rivas-de-Roca, R. (2020)

Ética periodística para una información con perspectiva de género

Revista Internacional de Investigación en Comunicación aDResearch ESIC. No 22 Vol 22

Monográfico especial, marzo 2020 · Págs. 82 a 97

https://doi.org/10.7263/adresic-022-04 
RESUMEN

\author{
Clasificación JEL: \\ D83, J16, L86 \\ Palabras clave: \\ Ética periodística, \\ medios de \\ comunicación, \\ códigos \\ deontológicos, \\ estudios de género, \\ mujer
}

Tema principal: El objetivo de este trabajo es la reflexión teórica sobre el valor que una nueva ética periodística puede jugar en el desarrollo de una información con perspectiva de género. La investigación aborda la función de los medios en la construcción social del género, a partir de la asimilación del paradigma de la responsabilidad social.

Desarrollo lógico del tema: La preocupación por el tratamiento informativo de estas cuestiones ha aumentado exponencialmente en los últimos años, de forma paralela al mayor grado de concienciación acerca de la relación entre hombres y mujeres. Tradicionalmente, las representaciones mediáticas del género habían contribuido al reforzamiento de arquetipos estereotipados. Frente a ello, las organizaciones periodísticas deben modificar su enfoque, aplicando una perspectiva de género. Esta responsabilidad en sentido amplio es en la que se basa nuestro estudio.

Punto de vista y aportaciones del autor: La principal aportación es la ampliación y categorización del concepto de ética periodística como central en la construcción de información con perspectiva de género. Esta ética comprende todas las fases del proceso de producción informativa, por lo que se articula como mecanismo para que los medios combatan los roles de género sexistas en su totalidad.

Repercusiones y conclusiones: Este artículo posee una repercusión sobre la representación social del género, derivada del desarrollo de una perspectiva periodística que contribuya a poner fin a arquetipos machistas. Además, nuestro planteamiento supone una enmienda a los principales códigos deontológicos de aplicación en España, desfasados en materia de igualdad de género. En cambio, algunos consejos audiovisuales autonómicos sí se encuentran en consonancia con la dimensión ética aquí expuesta. El periodismo, en tanto que profesión social, tiene que estar abierto a estas propuestas que, a su vez, contribuirían a satisfacer las necesidades informativas de las mujeres, habitualmente olvidadas por el carácter masculinizado de las empresas mediáticas.

\section{ABSTRACT}

\author{
JEL Classification: \\ D83, J16, L86 \\ Key words: \\ Ethics of \\ communication, \\ mass media, \\ deontological ethics, \\ gender studies, \\ woman
}

Main topic / subject: The objective of this work is to carry out a theoretical reflection on new communicative strategies, focused on journalistic ethics. This ethics would be useful for the setting-up of a journalism with gender perspective. The research addresses the role of the media in the social construction of gender, based on the social responsibility paradigm.

Logical development of the subject: In recent years, the concern for the informative treatment of these issues has increased exponentially, at the same time that a greater degree of awareness about the relationship between men and women has taken place. Traditionally, media representations of gender had contributed to the reinforcement of stereotyped archetypes. Against this, journalistic organizations must modify their approach, using a gender perspective. This responsibility in a broad sense is what our study is based on.

Author's point of view and contributions: Our main contribution is a new assessment of the concept of journalistic ethics, which becomes central to news with gender perspective. This ethics includes all phases of the information production process. Therefore, it is put forward as a tool for the media to fight sexist gender roles as a whole.

Implications and conclusions: The conclusions have impacts on the social representation of gender, triggered by the development of a journalistic perspective that helps to ending macho archetypes. In addition, our approach involves an amendment to the main ethical codes applied in Spain, outdated in gender equality. On the other hand, some regional audiovisual councils are aligned with our ethical dimension. Journalism, as a social profession, has to be open to these proposals that would contribute to meeting the informational needs of women, usually forgotten by the masculinized nature of media companies. 


\section{Introducción}

En los últimos años ha aumentado la preocupación por el tratamiento informativo que los medios de comunicación brindan a las cuestiones de género, de la mano de una mayor concienciación social sobre la desigualdad entre hombres y mujeres (Giménez Armentia y Berganza, 2009). Existe un consenso académico sobre el papel performativo de los medios de comunicación (Saperas, 1985; McQuail, 1994), en tanto en cuanto provocan efectos cognitivos sobre sus receptores. Los medios construyen la realidad mediada, relevante porque supone la representación pública de la actividad humana.

La representación mediática de las mujeres ha sido tradicionalmente sexista (Gallego Ayala, 2007; Menéndez Menéndez, 2007). Por ello, este artículo pretende reflexionar sobre la función que la ética periodística podría desempeñar en el desarrollo de una mirada de género, consciente de la desigualdad histórica entre los dos sexos. El objetivo es proporcionar una panorámica de la relación entre género y medios de comunicación, y en particular sobre cómo la ética puede contribuir a generar una información en igualdad.

Partimos de la premisa de que la igualdad entre hombres y mujeres requiere trascender informativamente la cobertura de temas de género, como las violencias machistas o la brecha salarial. Se antoja necesario construir una mirada de género que aplique esta perspectiva a todos los temas (Loscertales y Núñez, 2008). Si bien la difusión de estereotipos machistas en los medios es mucho menor que en el pasado, esto no ha impedido la pervivencia de férreas barreras de género en el mundo informativo (De Vuyst y Raeymaeckers, 2019).

Teniendo en cuenta lo anterior, se hace necesario emplear una perspectiva de género amplia para la ética periodística, que se centre también en parámetros como las redacciones, las empresas periodísticas o los índices de lectura (CaroGonzález García-Gordillo y Bezunartea-Valencia, 2014). La misión de esta investigación pasa por efectuar una revisión de la relación entre medios informativos y género, analizando las distintas herramientas profesionales al respecto, como los códigos deontológicos o la propia organización de las empresas mediáticas. Con ello buscamos categorizar una nueva dimensión de ética periodística que contribuya a un periodismo más responsable en la gestión de las cuestiones de género.

\section{Género y medios de comunicación}

Los medios de comunicación construyen socialmente la realidad, moldeando nuestra comprensión del mundo. En este sentido, la teoría del framing explica cómo la selección mediática de un determinado encuadre influye en la percepción del público sobre los hechos (Giménez Armentia y Berganza, 2009). Los estudios de género son muy críticos con el papel ejercido por el conjunto de medios de comunicación social en la representación de hombres y mujeres (Gallego Ayala, 2007; Mellor, 2012), considerando que han contribuido históricamente al mantenimiento de roles sexistas.

En primer lugar, debemos acotar científicamente la dimensión del término «género». El género es un constructo social, que alude a los roles asignados en función del sexo (Puleo, 2007). Históricamente, era habitual sostener que hombres y mujeres tenían predeterminadas unas funciones sociales según su sexo. En cambio, hoy en día decae esta concepción biológica frente a la constructivista, que valora al género «como una elección personal entre otras posibilidades del individuo» (Puleo, 2007, p. 13). Esta teoría del género procede de la reflexividad propia de la modernidad (Giddens, 1990), planteando las masculinidades 
y feminidades como elaboraciones culturales de los roles de hombres y mujeres en la sociedad.

La moderna teoría del género ha alterado la percepción de la realidad, generando actitudes críticas con respecto a los roles tradicionales. El estatus de género no solo afecta a la situación individual de los ciudadanos, sino también a numerosas variables socioculturales que determinan las prácticas cotidianas de la sociedad. Las relaciones sociales en desigualdad entre hombres y mujeres propician la aparición en las décadas de 1960 y 1970 del concepto político de género, que inicia una batalla institucional por acabar con los límites impuestos por la desigualdad (Friesem, 2016).

Los medios de comunicación social son actores privilegiados para el mantenimiento o el cambio social de los roles de género (WaterhouseWatson, 2016). Tradicionalmente, los medios, y en particular los no informativos vinculados al entretenimiento, han jugado un papel decisivo en la producción de los estereotipos sexistas, que hacen prevalecer la figura del hombre sobre la de la mujer. Dos casos llamativos son el cine (Arranz, 2010) y la publicidad (Yrache Jiménez, 2007). En ambos, la ideología machista ha calado más que en los actores informativos, debido a que el producto mediático se presenta como vehículo neutro de significado (Aparici y Barbas, 2010), diluyendo su componente ideológico.

Repasando las imágenes mediáticas asociadas al género, Gauntlett (2008) recoge la tendencia a mostrar a los hombres como asertivos, mientras que las mujeres desempeñan un rol pasivo. El primer estudio sistemático sobre la relación entre mujeres y medios de comunicación fue llevado a cabo por la norteamericana Gaye Tuchman (1978), obteniendo como resultado la aniquilación simbólica del sexo femenino en los medios, apareciendo infrarrepresentado. Su bajo peso se plasma en la menor presencia cuantitativa de las mujeres en los productos mediáticos. Además, en el plano cualitativo se las muestra como seres inferiores que no cuentan para los asuntos importantes.

Aunque es cierto que la situación ha cambiado en las tres últimas décadas, con la difusión de arquetipos más igualitarios, permanece un cierto legado sexista (Lauzen, Dozier y Nora Horan, 2008). La mujer es representada como más independiente, acorde a su nueva posición social, pero sigue teniendo menos voz en las cuestiones de relevancia, como la política (Thumin, 2004). Existe asimismo un peligro de apropiación cultural del feminismo, puesto que los derechos de los grupos marginalizados, como las mujeres, se han convertido en parte de la cultura popular (Gauntlett, 2008).

En cualquier caso, parece claro que desde los 90 se incrementan los roles igualitarios y no estereotipados, tanto en los productos mediáticos factuales como en los de ficción. La pregunta es si esto implica necesariamente una mejor representación. Los roles de género transmitidos por los medios resultan más complejos que nunca antes, si bien perviven ciertos estereotipos difundidos ahora de manera difusa, fruto de rutinas profesionales muchas veces imperceptibles (Gauntlett, 2008). Esto obliga a aplicar una perspectiva de género a todas las fases de producción mediada, siendo necesaria una alfabetización de los actores involucrados de la cual la industria todavía no ha adquirido conciencia (Friesem, 2016).

\subsection{Los medios de comunicación informativos}

En la esfera estrictamente informativa, los medios constituyen fuente nutricia del imaginario colectivo de género (O’Brien, 2018). El lenguaje es una construcción social, que revela las categorías simbólicas utilizadas por la sociedad. La información emplea una modalidad singular del 
lenguaje, el llamado lenguaje periodístico, que establece una distribución de lo que existe (Gallego Ayala, 2007).

Mediante esa organización de la realidad, los medios sancionan lo que está permitido socialmente y lo que no, efectuando una representación pública de las actividades humanas (Saperas, 1985). El lenguaje para representar el género ha sido tradicionalmente asimétrico. Las mujeres aparecen en el discurso público como objeto observado, mientras que los hombres se presentan como agente activo (Gallego Ayala, 2007). Al igual que en los productos no factuales, tanto la cantidad como la calidad de las alusiones por género denotan asimetría.

Los medios, en tanto que instituciones sociales, gestionan poder (Scheufele, 2000; Scheafer, 2001). La prensa escrita ha sido considerada tradicionalmente como referencia para el resto de medios de comunicación, que se dedicaban a replicar sus contenidos. En los periódicos resulta más notable la construcción de un nosotros (hombres) frente a un ellas, independientemente del lector (Gallego Ayala, 2007). Por tanto, la reproducción social de la desigualdad entre hombres y mujeres cuenta con un aliado en los medios de comunicación, que actúan como subsistema de producción simbólica que alimenta la dominación masculina (Callejo, 2000).

Si bien académicamente las Ciencias de la Información suponen una de las áreas más feminizadas, las redacciones mantienen rutinas de funcionamiento machistas que dificultan la consecución de una igualdad real (De Vuyst y Raeymaeckers, 2019). De hecho, conceptos como feminismo generan un cierto rechazo en este ámbito, evidenciando su desconocimiento sobre las cuestiones de género (North, 2009). Las razones para esta situación pueden hallarse en el carácter históricamente masculino de la profesión, con horarios que aún hoy en día hacen imposible la conciliación, así como en el hecho de que sean varones los que copan los puestos de decisión.

En un estudio diacrónico del análisis informativo del issue «women», Gómez Patiño (2014) aprecia que el tratamiento de las cuestiones feministas en los principales periódicos españoles es puramente circunstancial, vinculado a efemérides como la celebración del Día Internacional de la Mujer el 8 de marzo. Además, la agenda política posee influencia sobre la periodística, de tal forma que el planteamiento informativo varía según el gobierno de turno, a la vez que en función de la adscripción ideológica de diario. El issue «women» se revela como un tema débil, fluctuante según la circunstancia.

Por otro lado, un asunto de género controvertido para los medios informativos es la cobertura de las violencias machistas. La representación de la violencia contra las mujeres no es solo importante por la capacidad performativa de los medios, que moldean nuestra capacidad de entender el mundo, sino también porque afecta a los estilos de vida. Las noticias advierten a las mujeres sobre acciones inseguras, influyendo en sus pequeñas decisiones cotidianas como la vestimenta o la hora de regreso a casa (Zurbano-Berenguer y GarcíaGordillo, 2017). Los medios dan cuenta de cómo la sociedad interpreta los actos de violencia machista, delimitando lo que es aceptable. Históricamente, esta construcción simbólica ha tenido un fuerte componente machista, propiciando un sentimiento de culpa en las mujeres objeto de agresiones, fruto de ideas latentes en los mensajes mediáticos (Vallejo, 2005).

La dimensión social de las noticias sobre violencia contra las mujeres es mayor que en otras cuestiones de género, ya que estas cuestiones aluden a un grupo de máxima vulnerabilidad. La ética periodística exige en estos casos de una 
especial diligencia y sensibilidad a la hora de informar. Una mala praxis generará aún más dolor a un colectivo que atraviesa una situación especialmente difícil (Pérez Fuentes, 2004). Por ello, se reclama que la información de este tipo se ampare en las tesis de teoría de la responsabilidad social de los medios (Fernández y Noblejas, 2011), que hunde sus raíces en la tradición norteamericana (Siebert, Peterson y Schramm, 1956).

\section{Hacia un periodismo con perspectiva de género}

\subsection{Empresas periodísticas "feministas»}

Hasta ahora se ha reflexionado en torno a la dimensión simbólica de los medios en la construcción de los roles de género, planteando un cambio en las actuaciones de los mismos en pro de una sociedad igualitaria. En este sentido, resulta necesario definir qué significa que los medios empleen la perspectiva de género anteriormente citada. Recogemos para ello la propuesta teórica de Menéndez Menéndez, que sostiene que «la perspectiva de género es un modelo de interpretación de la realidad que permite reconocer la desigualdad histórica que soportan las mujeres y visibilizar las aportaciones de estas a la sociedad» (2007, p. 155).

En el ámbito del periodismo, la perspectiva de género implica desarrollar una información que reconozca la existencia de estereotipos y visibilice a las mujeres, tratando de construir mediáticamente una sociedad no sexista. La solución para ello pasaría por cambiar la representación que los medios hacen de las mujeres y aumentar los canales de participación para ellas y su presencia en puestos con capacidad de decisión, a lo que se uniría la elaboración de discursos informativos que fomenten la eliminación del sexismo en la sociedad (Menéndez Menéndez, 2007).

Medidas como la intervención sobre la composición de los puestos de mando evidencian que la perspectiva de género actual trasciende del mero tratamiento informativo, abarcando también otras cuestiones como la dinámica de la redacción o la naturaleza de la empresa informativa (Callejo, 2000; Caro-González, 2011). Se trata, por tanto, de una responsabilidad social en sentido amplio, cuyo éxito radicaría en ir dirigida a las distintas fases de producción del producto mediático (Arranz, 2010).

En el contexto de esta perspectiva de género, podríamos acuñar el término de empresas periodísticas «feministas» para referir a aquellos medios que funcionan de acuerdo a una lógica igualitaria en la relación entre hombres y mujeres. Esto supone aplicar la perspectiva de género a las empresas informativas, algo necesario dado que existen estudios que señalan que los medios con mayores audiencias femeninas son aquellos que cuentan con una mayor presencia de mujeres en su equipo directivo y en su redacción (Caro-González, García-Gordillo y Bezunartea-Valencia, 2014).

La investigación realizada por Caro-González, García-Gordillo y Bezunartea-Valencia (2014) muestra una correlación entre la presencia femenina en la cúpula directiva de los medios y el número de lectoras. La vinculación con el género es total en todas las fases del proceso productivo: los periódicos con más mujeres directivas cuentan con más mujeres periodistas, que a su vez recurren a fuentes femeninas y alcanzan a un mayor volumen de lectoras. Estos datos resultan de interés, puesto que la mujer ha tenido siempre una escasa relación con la prensa escrita, lo que encontraría aquí una explicación.

El número de mujeres lectoras de prensa se mantiene inferior al de hombres en España. De acuerdo a los datos de la encuesta de Hábitos de Lectura, en 2017 el 82 \% de los hombres leía una vez al trimestre prensa, ya sea en formato digital o en papel, frente al $67 \%$ de las mujeres. 
Sin embargo, la cifra de mujeres estudiantes en las facultades de Periodismo lleva siendo mucho más elevada que la presencia de hombres desde la década de 1990 (Callejo, 2000). Este peso académico tampoco tiene una correspondencia en las más altas esferas de la actividad profesional, donde la presencia de mujeres era anecdótica hasta principios del siglo XXI.

Es cierto que ha habido grandes cambios en este sentido en las décadas recientes. Desde 2002, el porcentaje de mujeres directivas en periódicos de información general y televisiones, públicas y privadas, se ha incrementado exponencialmente. Se ha pasado de la ausencia total a lograr una presencia de mujeres superior al 20 \% en España (APM, 2018). Para ello ha sido fundamental la mayor concienciación social, acompañada de la Ley Orgánica 3/2007, de 22 de marzo, para la igualdad efectiva de mujeres y hombres, que promovió la igualdad en los ámbitos directivos.

Sin embargo, la mejora conseguida se revela insuficiente: la disparidad del número de lectoras y directivas con respecto a sus homólogos masculinos evidencian un problema de desigualdad de género. Esta escasa presencia provoca que la visión del más del 50 \% de la población se encuentre informativamente ausente, una simplificación de la realidad que atenta contra los valores democráticos. Una mayor representación de las mujeres en estas empresas provocaría un cambio de la agenda mediática, incorporando cuestiones femeninas hasta ahora olvidadas que, además, contribuirían a desterrar estereotipos sexistas (Loscertales y Núñez, 2008).

Por tanto, los medios deben ofrecer contenidos que satisfagan las necesidades informativas de las mujeres. Los valores femeninos se suelen asociar a la empatía y al pragmatismo (Bezunartea-Valencia, García-Gordillo y Rodríguez Rey, 2012), aspectos olvidados en los diarios en pro de una imagen competitiva de la sociedad. Asimismo, en los medios el hombre aparece vinculado con el rol de autoridad. Todo esto provoca que sea difícil para las mujeres sentirse partícipes de la percepción del mundo representada por los medios de comunicación (Armstrong, 2004).

Existen otras barreras que merman el acceso de la mujer a puestos de relevancia en la empresa periodística. La excesiva preocupación organizativa, fruto de una visión economicista de los medios, limita la puesta en marcha de cambios en las rutinas profesionales que favorezcan a las mujeres (Caro-González, 2011). Esto entronca con el hecho de que el periodismo sea una profesión fuertemente mitificada. Uno de los principales mitos pasa por el individualismo: el periodista es considerado un profesional liberal, que está por encima de la realidad, colabora poco con sus compañeros y no admite injerencias externas (Callejo, 2010). Esta visión de la profesión deja la introducción de cambios a expensas de la arbitrariedad, lo que obstaculiza un nuevo rol de las mujeres.

El gran incremento del número de periodistas mujeres en los últimos años ha generado en algunos académicos un alto nivel de optimismo, apuntando incluso a la pronta desaparición de los estereotipos sexistas en el ámbito informativo (Byerly, 2013). Sin embargo, estudios recientes, como el efectuado por De Vuyst y Raeymaeckers (2019) relativo a los principales periódicos belgas, reflejan la existencia de barreras consolidadas para la igualdad de género, con solo un $30 \%$ de presencia femenina. Como se había anticipado, la escasa flexibilidad de las redacciones para la conciliación familiar -las mujeres aún sostienen la mayor parte de la carga en el hogar-, unida ahora a la vida errante de los cada vez más habituales freelances o el uso continuo de nuevas 
tecnologías, que presentan un déficit por sexo favorable a los hombres, coartan el desarrollo de una igualdad real.

Según datos del Global Media Monitoring Project, entre 2005 y 2015 la cifra de mujeres se situó en el $37 \%$ sobre el total de periodistas registrados a nivel mundial (GMMP, 2015). En cambio, este porcentaje se reduce a medida que se avanza en la escala laboral. Las mujeres solo representan el $28 \%$ en puestos intermedios y no alcanzan el $16 \%$ en cargos directivos (GMMP, 2015), evidenciando la existencia de un techo de cristal. Esta brecha es también generacional, puesto que hay estudios que señalan que el número de mujeres es menor en franjas de edad elevadas (De Vuyst y Raeymaeckers, 2019).

\subsection{Códigos deontológicos en el tratamiento informativo de las mujeres}

La actividad periodística se enfrenta a enormes dificultades para su regulación, debido a las características intrínsecas de la profesión. El derecho de la información está recogido en el mundo occidental como un precepto abierto; todo lo que suponga establecer normas punitivas se vincula con la censura propia de las dictaduras (Aznar Gómez, 2005). En consecuencia, solo se produce autorregulación a través de códigos deontológicos, lo que explica el escaso éxito de los colegios de periodistas.

Salvo excepciones en países como Reino Unido, las normas deontológicas tienen un papel puramente referencial, mucho menor que el que se observa en otras profesiones. Por ello, depende de cada medio la aplicación de estos mínimos estándares, que en ningún caso ponen en cuestión aspectos como la propiedad o la estructura interna de la empresa informativa. La inspiración moral de estos códigos procede de la ética periodística, un ideal kantiano referido al buen hacer de la actividad informativa para la mejora de la sociedad (Pérez Fuentes, 2004).

Esta porosidad regulatoria lleva a que sea difícil calibrar la actividad de los medios. Los colegios de periodistas han tenido escasa implantación, dada la imposibilidad de fijar requisitos de titulación mínimos para acceder a una profesión de carácter abierto. En el caso de España, la figura de mayor éxito son las asociaciones de periodistas, unas organizaciones profesionales que velan por los intereses de sus representados y establecen normas de autocontrol deontológico.

En virtud de lo anterior, este trabajo efectúa un repaso de las referencias a la igualdad de género en los principales códigos deontológicos que tienen aplicación en España, incluyendo también los libros de estilo de los principales diarios de nuestro país. Estos libros suponen un mecanismo de autorregulación clásico de los periódicos que comprende desde la dimensión ética al estilo de redacción. Desde un punto de vista universal, los Principios Internacionales de Ética Profesional del Periodismo, aprobados por la UNESCO en 1983, no aluden directamente a la cuestión de género. Sí que recogen propuestas abiertas en las que se podría enmarcar esta temática, como el Principio III de «responsabilidad social del periodista» y el Principio $\mathrm{X}$ de «promoción de un nuevo orden mundial de la información y la comunicación».

La responsabilidad social del periodista, en consonancia con la teoría del mismo nombre, se vincula a la percepción de la información como un bien social, no como un producto. Esto significa que el periodista ejerce una responsabilidad sobre la información transmitida, debiendo ser consciente de sus implicaciones y de la reproducción de los estereotipos sexistas. Además, la mención a un nuevo orden mundial de la información incorpora la variante de generar un 
sistema informativo a nivel global que fomente la democratización y el bienestar, a partir de la inclusión de los grupos marginalizados. Aunque esta propuesta, formulada por Sean MacBride en 1980, se refiere a un nuevo tipo de relaciones internacionales, esto solo sería posible con la participación del colectivo más discriminado de la historia: las mujeres.

En cualquier caso, en el texto de la UNESCO no aparece ninguna alusión directa a la desigualdad de género. En cambio, a escala europea el Código Deontológico Europeo de la Profesión Periodística del Consejo de Europa sí contiene referencias al respecto. En el artículo 33, localizado en el apartado «Situaciones de conflicto y casos de protección especial», se comenta brevemente que los medios de comunicación tienen la obligación moral de rechazar «toda discriminación por razón de cultura, sexo o religión». Por su parte, en las recomendaciones al Comité de Ministros del Consejo de Europa la igualdad de género aparece de forma más clara, citada en relación a los medios públicos.

Según ese documento, los medios de comunicación públicos deben promover la igualdad de género en sus informaciones. Se trata de un avance frente al texto de la UNESCO, que carece de referencias al género. Sin embargo, la acción propuesta por el Consejo de Europa se restringe únicamente a los medios de naturaleza pública, obviando el gran mercado de información privada. Hay que tener en cuenta que el código europeo data de 1993, fecha en la que todavía se consideraba una intromisión plantear desde las esferas públicas preceptos deontológicos para los organismos privados dedicados al periodismo.

En España, la FAPE (Federación de Asociaciones de Periodistas de España), la mayor organización de periodistas de nuestro país que aglutina a las distintas asociaciones provinciales, consagra en el Principio 7 de su Código Deontológico que «el periodista extremará su celo profesional en el respeto a los derechos de los más débiles y los discriminados. Por ello, debe mantener una especial sensibilidad en los casos de informaciones u opiniones de contenido eventualmente discriminatorio». La recomendación se completa señalando que el periodista debe abstenerse de realizar comentarios despectivos en relación a la raza, color, religión, origen social o sexo de la persona.

Si bien el código de la FAPE cita la necesidad de no emplear el sexo de una persona con fines discriminatorios, incluyendo este caso en los de especial protección, su referencia al género es totalmente accesoria y unida a otras categorías sociales. El documento de esta organización data de 1993, pero fue actualizado en 2017, lo que hace más llamativa la falta de alusión a la igualdad de género como tal. Es en los códigos de los consejos audiovisuales -organismos autonómicos autorreguladores del mercado audiovisual- donde la función social de los medios en aras de la igualdad real queda más manifiesta.

Un ejemplo de lo anterior es el Código deontológico de los miembros del Consejo Audiovisual de Andalucía. En el apartado «Principios básicos» ya se hace mención a la necesidad de combatir la desigualdad de género, mientras que en los «Principios éticos» se desarrolla cómo ha de acontecer su actuación: «(los miembros del Consejo Audiovisual de Andalucía) velarán, en el ámbito de sus competencias, por promover la efectiva igualdad entre hombres y mujeres, y trabajarán para remover los obstáculos que puedan dificultarla».

También resultan de interés los conocidos como libros de estilo, manuales de redacción periodística habituales en los diarios españoles que dan cuenta asimismo de cuestiones éticas a valorar en la elaboración de la información. Un breve análisis de los libros de estilo de los dos 
periódicos generalistas más leídos en nuestro país -El País y El Mundo- muestra una inexistencia total de preceptos sobre igualdad de género, machismo o estereotipos sexistas. La perspectiva de género se halla completamente ausente de estos documentos, al menos de modo visible. Ni siquiera se indica el empleo de términos genéricos o el uso de masculino aplicado indistintamente en género masculino y femenino, como es habitual en organismos públicos y privados comprometidos con la igualdad de género.

En conclusión, el panorama de códigos deontológicos a tres niveles -internacional, europeo y nacional- que rige en nuestro país no parece proclive a valorar los temas de género como una cuestión objeto de la ética periodística. Las menciones son mayores cuanto más se acerca el ámbito del código a la proximidad geográfica, pero en ningún caso se formula el género como un problema en sí mismo. A este respecto debemos tener en cuenta la antigüedad de los códigos, que fueron elaborados en los años 80 y 90 . Las actualizaciones realizadas en ellos, como la efectuada sobre el Código Deontológico de la FAPE, no han paliado este déficit, algo que se repite en los libros de estilo de los dos periódicos generalistas más leídos.

Frente a la falta de conciencia sobre género evidenciada por los principales códigos deontológicos y libros de estilo, los consejos audiovisuales autonómicos desarrollan códigos que promueven la igualdad efectiva de hombres y mujeres desde la acción de los medios de comunicación. Así sucede tanto con el Consell de l'Audiovisual de Catalunya (CAC), creado en 1997, como con el Consejo Audiovisual de Andalucía, que data de 2005. Por tanto, formalmente los consejos audiovisuales constituyen una excepción en la preocupación deontológica sobre la influencia de los medios en los roles de género.

\section{La ética periodística como propuesta profesional}

Los códigos deontológicos se han demostrado insuficientes, pero la mera existencia de estos preceptos autorreguladores sugiere la vigencia del concepto de responsabilidad social en nuestras democracias (Norris, 2000; Mazzoleni, 2017), central para promover un periodismo con perspectiva de género. La responsabilidad social implica que tanto el profesional de la información como el medio de comunicación actuarán en todas las circunstancias en conformidad con su propia conciencia ética, tal y como recogen los Principios Internacionales de Ética Profesional del Periodismo de la UNESCO.

En lo referente al tratamiento informativo, son muchas las noticias que tienen como tema principal la violencia contra las mujeres, en sus distintas variantes de maltrato físico, sexual o trata de blancas (Berganza, 2003). Como aspecto positivo cabe destacar el abandono de expresiones como «crimen pasional»o «violencia doméstica», recurriéndose ahora a terminologías que permiten enfocar esta realidad como un problema social (Martín, Armentia-Vizuete y Caminos, 2012). Se superponen en el discurso periodístico conceptos como «violencia machista» o «de género», favoreciendo una identificación conceptual con la gravedad del problema.

El empleo de un lenguaje libre de sexismos resulta fundamental para la construcción de un periodismo con perspectiva de género, algo que también ha sido estudiado en América Latina (Chaher y Santoro, 2007). En este sentido, en los últimos tiempos está adquiriendo notoriedad en los medios el término «violencias machistas», que engloba todas las acciones forzosas dirigidas a las mujeres, desde las agresiones sexuales a la trata de blancas. La fórmula «violencias machistas» constituye una buena práctica a imitar, en tanto 
en cuanto contribuye a comprender el carácter estructural de la violencia hacia las mujeres. Lo importante no es el efecto inmediato de la información, sino la capacidad a largo de plazo de influir sobre el conocimiento y la sensibilidad con esta materia (Carballido, 2009).

Como hemos visto, la ciudadanía basa su conocimiento sobre cuestiones de género, como las violencias machistas, en la información proporcionada por los medios de comunicación. Su capacidad de influencia radica en dos elementos: el grado de conocimiento social de la desigualdad de género y la conceptualización de este problema a través de la conformación de los imaginarios colectivos (Israel Garzón, 2006). Estas dos áreas suponen espacios de actuación para una ética periodística contraria al machismo.

En el tratamiento de la información acerca de cuestiones de género sería interesante emplear la llamada «ponderación de bienes», un mecanismo profesional de autorregulación que consta de tres elementos: 1) veracidad y relevancia pública de la información; 2) «principio de proporcionalidad» de lo publicado, es decir, si lo dado a conocer era necesario y se ha hecho público intentando vulnerar lo menos posible los derechos de los grupos sensibles, en este caso las mujeres; 3) doctrina del reportaje neutral, que consiste en dejar espacio periodístico para opiniones contrapuestas, siempre y cuando no atenten contra los derechos de las mujeres (Rodríguez Gómez, 2014).

En España, el artículo 20. 1) de la Constitución, que recoge el derecho a la información, suele entrar en confrontación con el artículo 18, dedicado a la protección al honor, la intimidad y la propia imagen. A lo largo de su historia, el Tribunal Constitucional ha concedido «prevalencia al artículo 20.1 d) sobre el artículo 18.1», en lo que es una muestra de que pocas intromisiones en la intimidad amparan la suspensión de la liber- tad de información (Rodríguez Gómez, 2014, p. 1212). No obstante, el interés público no puede ser óbice para difundir noticias que promuevan la desinformación sobre los temas de género. En este caso, resulta aún más pertinente aplicar el «principio de proporcionalidad», tratando siempre de fomentar el desarrollo de una ciudadanía consciente acerca de un problema que atenta contra los valores básicos de igualdad.

Más allá de la información publicada, la complejidad de las cuestiones de género, y en particular de las violencias machistas, requiere de una interpretación periodística que tenga en cuenta la interrelación de sus múltiples dimensiones (Laguna, 2009). Su intrincada naturaleza exige además una acción conjunta de los responsables políticos, académicos y sociales, encaminada como objetivo final hacia la erradicación de la desigualdad de género. La primera medida mediática pasa por realizar análisis multidisciplinares del problema, que construyan un rechazo social a la desigualdad de tipo multinivel, tanto en el ámbito externo como interno del medio.

La literatura ha demostrado la vigencia de factores estructurales que mantienen los roles de género en el ámbito periodístico. El qué -la mujer como objeto informativo- condiciona inexorablemente el cómo (García-Gordillo, 2011; Friesem, 2016), dado que se sigue una lógica androcéntrica en la información, aún más acentuada en otros productos mediáticos como la publicidad o las revistas. Frente a esta situación, abogamos por la configuración de una ética periodística amplia, que fomente la incorporación de las mujeres en los puestos de mayor responsabilidad informativa, así como la agregación al periodismo de valores asociados tradicionalmente a las feminidades. El oficio adolece de estar excesivamente masculinizado en sus dinámicas profesionales. 
Algunas de las recomendaciones para estas organizaciones periodísticas son: el establecimiento de rutinas laborales más flexibles, que faciliten la conciliación de la vida familiar -a la espera de la igualdad real en el cuidado de los hijos-; una paulatina introducción de las nuevas tecnologías, que no perjudique a las mujeres, discriminadas socialmente desde la niñez en el uso de la tecnología; o la progresiva deconstrucción de mitos de la profesión periodística, como el individualismo, que chocan con la introducción de una visión femenina.

En los últimos años se produce un cierto optimismo sobre las posibilidades de Internet en la difusión de noticias feministas, efectuando una representación de la esfera pública que deconstruye los discursos. También emergen críticas a la capacidad de la Red para fomentar estereotipos machistas, muy visibles en sectores como el publicitario (Steiner, 2005). Estos nuevos planteamientos permearían la lógica de los medios. El problema radica en que las tecnologías pueden reproducir las desigualdades existentes, manteniendo una estrategia puramente economicista.

\section{Conclusiones}

En este documento se ha tratado la labor de los medios en la construcción social del género, reflexionando acerca de la necesidad de articular la ética periodística como clave para una información con perspectiva de género. Esta voluntad nace de la vigencia del paradigma de la responsabilidad social de los medios de comunicación, asimilado por emisores y receptores del periodismo. La literatura científica cuenta con aproximaciones que invitan a promover una responsabilidad periodística en sentido amplio, tesis de la que parte esta investigación. El carácter estructural de la desigualdad de género se halla detrás de esta propuesta.
A continuación, resumimos en cincos puntos las principales conclusiones del artículo:

1. Los medios de comunicación social tienen la capacidad de clasificar y construir los grupos sociales. Esto es significativo para las relaciones de género, ya que suponen relaciones sociales atravesadas por las diferencias de poder.

2. Los medios informativos han contribuido históricamente al mantenimiento y reproducción de los estereotipos sexistas. Para revertir esta dinámica, las organizaciones periodísticas y sus profesionales deben ampliar la mirada, lo que entraña aplicar la perspectiva de género. Este enfoque permea el conjunto de las noticias, tratando de percibir en ellas el problema histórico de la desigualdad entre hombres y mujeres para darlo a conocer, a la vez que se conceptualiza como un conflicto social.

3. La perspectiva de género debería estar presente en todas las fases de la producción mediática. No se cumplen las necesidades informativas de las mujeres porque las empresas periodísticas no están construidas desde una óptica femenina, existiendo una correlación en este proceso. A través de la promulgación de rutinas profesionales menos masculinizadas se podría alcanzar un mayor número de mujeres en puestos directivos de los medios $\mathrm{y}$, por ende, de lectoras.

4. Los principales códigos deontológicos de aplicación en España -Principios Internacionales de Ética Profesional del Periodismo, Código Deontológico Europeo de la Profesión Periodística y Código Deontológico de la FAPE- se encuentran desfasados en el ámbito de la igualdad de género. Tampoco cuentan con información al respecto los libros de estilo de El País y El Mundo. Todo ello evidencia un problema de moderniza- 
ción en los preceptos autorreguladores. La excepción la constituyen los más recientes consejos audiovisuales autonómicos, como los de Cataluña o Andalucía, que recogen la igualdad de género como máxima a perseguir por la acción de sus medios.

5. Se ha conseguido redefinir la ética periodística, entendiéndola aquí como respuesta totalizadora para construir medios e informaciones que promuevan el fin de los roles de género sexistas en todas sus actuaciones. Por tanto, la aplicación de esta ética se amplía a todas las fases del proceso de producción mediática. El componente social del periodismo obliga a poner el foco en el desarrollo de esta ética responsable, que trabaja para la igualdad real entre hombres y mujeres. No tomar partido en esta materia iría en contra de los principios democráticos de la profesión.

El inconveniente de esta nueva ética es que, como todo elemento autorregulador, depende de la buena voluntad de las personas que se dedican a la actividad periodística. No parece plausible aspirar por el momento a algo superior a la autorregulación en las sociedades democráticas. Sin embargo, cuanto más se visibilice la perspectiva de género, cuanto mayor sea el número de referencias que se hagan a ella en los medios o en los propios códigos deontológicos, más plausible es que emisores y receptores la conozcan, para después interiorizarla como enfoque a emplear.

Independientemente de la tiranía o no que ejerzan los medios sobre sus contratados, el periodismo es una profesión de valores, lo que puede favorecer la toma de conciencia sobre la desigualdad de género. Muchas rutinas profesionales machistas han sido adquiridas de forma totalmente inconsciente, lo que abre la puerta a que una mayor reflexividad sobre el quehacer periodístico logre promover una información más «feminista» $y$, por ende, más acorde a los valores democráticos. 


\section{Bibliográfía}

Aparici, R. \& Barbas, A. (2010). Estereotipos, ideología y representación mediática en la construcción de los relatos. En Aparici, R. (Coord.). La construcción de la realidad en los medios de comunicación (pp. 30-58). Madrid: UNED.

Armstrong, C. L. (2004). The influence of reporter gender on source selection in newspaper history. Journalism and Mass Communication Quarterly, 81(1), 139-154.

Arranz, F. (2010). La igualdad de género en la práctica cinematográfica española. En Arranz, F. (Ed.). Cine y género en España (pp. 17-68). Madrid: Cátedra, Colección Feminismos.

Asociación de la Prensa de Madrid (2018). Informe Anual de la Profesión Periodística. Madrid: APM.

Aznar Gómez, H. (2005). Ética de la comunicación y nuevos retos sociales: códigos y recomendaciones para los medios. Barcelona: Paidós.

Berganza, R. (2003). Media construction of violence against women: a framing approach. Communication \& Society, 16(2), 9-32.

Bezunartea-Valencia, O., García-Gordillo, M. \& RodríguezRey, A. (2012). La mujer como cargo y como fuente en la prensa escrita. La paridad no llega a las noticias. Ámbitos: Revista Internacional de Comunicación, 21, 233-256.

Byerly, C. M. (2013). The Palgrave International Handbook of Women and Journalism. Nueva York: Palgrave Macmillan.

Callejo, J. (2000). Medios, género y poder. En García de Cortázar, M. y García de León, M. A. (Eds.). Profesionales del periodismo. Hombres y mujeres en los medios de comunicación (pp. 1-29). Madrid: Centro de Investigaciones Sociológicas. Carballido, P. (2009). Medios de comunicación social y violencia de género. Una revisión desde la teoría del framing. En Bernardo, J. M., Martínez, E. \& Montiel, G. (Coords.). Retos de la Comunicación ante la Violencia de género. Marco jurídico, discurso mediático y compromiso social (pp. 157174). Barcelona: Tirant Lo Blanch.

Caro-González, F. (2011). La satisfacción de las necesidades informativas de las mujeres. Análisis de la empresa periodística desde la perspectiva de género (Memoria de investigación INVM PR04107). Extraído el 06.12.2019 desde http://bit. ly/1BiNPli
Caro-González, F., García-Gordillo, M. \& Bezunartea-Valencia, O. (2014). Women and the press: why so few women read newspapers. Estudios sobre el Mensaje Periodístico, 20(2), 9871002. DOI: https://doi.org/10.5209/rev_ ESMP.2014.v20.n2.47045

Chaher, S. \& Santoro, S. (comps.) (2007). Las palabras tienen sexo: introducción a un periodismo con perspectiva de género. Buenos Aires: Artemisa Comunicación Ediciones.

Consejo Audiovisual de Andalucía (2004). Código deontológico de los miembros del Consejo Audiovisual de Andalucía. Extraído el 06.12.2019 desde http://www.consejoaudiovisualdeandalucia.es/sites/default/files/codigo_deontologico_de_los_miembros_del_caa.pdf

Consejo de Europa (1993). Código Deontológico Europeo de la Profesión Periodística. Extraído el 06.12.2019 desde http:// www.asociacionprensa.org/es/images/Codigo_Deontologico_Europeo_de_la_Profesion_Periodistica.pdf

De Vuyst, S. \& Raeymaeckers, K. (2019). Gender as a multilayered issue in journalism: A multi-method approach to studying barriers sustaining gender inequality in Belgian newsrooms. European Journal of Women's Studies, 26(1), 23-38. DOI: https://doi.org/10.1177/1350506817729856

Federación de Gremios de Editores de España (2018). Hábitos de Lectura y Compra de Libros en España 2017. Extraído el 06.12.2019 desde https://www.federacioneditores.org/ img/documentos/HabitosLecturaCompraLibros2017.pdf

Federación de Asociaciones de Periodistas de España (1993). Código Deontológico de la FAPE. Extraído el 06.12.2019 desde http://fape.es/home/codigo-deontologico/ Fernández, A. \& Noblejas, M. (2011). Cómo informar sobre violencia contra la mujer en las relaciones de pareja. Madrid: Escuela de Periodismo y Comunicación.

Friesem, E. (2016). Drawing on Media Studies, Gender Studies, and Media Literacy Education to Develop an Interdisciplinary Approach to Media and Gender Classes. Journal of Communication Inquiry, 40(4), 370-390. DOI: https://doi. org/10.1177/0196859916656837

Gallego Ayala, J. (2007). Lenguaje periodístico y discriminación de género. En Plaza, J. y Delgado C. (Eds.). Género y Comunicación (pp. 49-71). Madrid: Fundamentos.

García-Gordillo, M. (2011). Mujeres, medios e información o de cómo el qué condiciona el cómo. En Mancinas, R. y Nogales, A. (Eds.). La mujer en el espejo mediático: el medio- 
trato femenino (pp. 25-40). Sevilla: Asociación Universitaria Comunicación y Cultura (AUCC).

Gauntlett, D. (2008). Media, Gender and Identity. An introduction. Londres y Nueva York: Routledge.

Giddens, A. (1990). The Consequences of Modernity. Stanford: Stanford University Press.

Giménez Armentia, P. \& Berganza, R. (2009). Género y Medios de Comunicación. Un análisis desde la Objetividad y la Teoría del Framing. Madrid: Editorial Fragua.

GMMP (Global Media Monitoring Project) (2015). Who Makes the News? Londres: World Association for Christian Communication.

Gómez Patiño, M. (2014). La influencia de la agenda setting: análisis comparado del tratamiento del issue 'mujer' en la prensa española (2007-2012). Estudios sobre el Mensaje Periodístico, 20(2), 1103-1120.

Israel Garzón, E. (2006). Representación informativa de la violencia contra las mujeres. Tendencias, recomendaciones y propuestas para periodistas sociales. UNIrevista, 3, 1-20.

Laguna, R. (2009). El impacto social de la violencia de género a través de los medios de comunicación. Diez años de evolución del tratamiento periodístico en la lucha contra la violencia sobre la mujer. En Bernardo, J. M., Martínez, E. y Montiel, G. (Coords.). Retos de la Comunicación ante la Violencia de género. Marco jurídico, discurso mediático y compromiso social (pp. 295-304). Barcelona: Tirant Lo Blanch.

Lauzen, M. M., Dozier, D. M. \& Nora Horan M. A. (2008). Constructing Gender Stereotypes Through Social Roles in Prime-Time Television. Journal of Broadcasting \& Electronic Media, 52(2), 200-214. DOI: https://doi.org/10.1080/08838 150801991971

Loscertales, F. \& Núñez, T. (2008). Los medios de comunicación con mirada de género. Sevilla: Instituto Andaluz de la Mujer.

McQuail, D. (2000) [1994]. McQuail's mass communication theory. Londres: Sage.

Martín, F., Armentia-Vizuete, J. I. \& Caminos, J. M. (2012). El tratamiento informativo de las víctimas de violencia de género en Euskadi: Deia, El Correo, El País y Gara (20022009). Comunicación y Sociedad, 24(2), 412-435.

Mazzoleni, G. (2017). Changes in Contemporary Communication Ecosystems Ask for a «New Look» at the Concept of Mediatisation. Javnost - The Public, 24(2), 136-145. DOI: https://doi.org/10.1080/13183222.2017.1290743
Mellor, N. (2012). Hearts of Steel: Female journalists reflecting on their professional ethics. Feminist Media Studies, 12(2), 180-194. DOI: https://doi.org/10.1080/14680777.2 011.597099

Menéndez Menéndez, M. I. (2007). Claves prácticas para la elaboración y revisión de textos periodísticos desde la perspectiva de género. En Plaza, J. y Delgado C. (Eds.). Género y Comunicación (pp. 149-168). Madrid: Fundamentos.

Norris, P. (2000). A Virtuous Circle. Political Communications in Postindustrial Societies. Cambridge: Cambridge University Press.

North, L. (2009). Rejecting the 'F-word' How 'feminism' and 'feminists' are understood in the newsroom. Journalism, 10(6), 739-757. DOI: https://doi.org/10.1177/146488 4909344479

Pérez Fuentes, J. (2004). Ética periodística: principios, códigos deontológicos y normas complementarias. Bilbao: Servicio Editorial de la Universidad del País Vasco.

Puleo, A. H. (2007). Introducción al concepto de género. En Plaza, J. y Delgado C. (Eds.). Género y Comunicación (pp. 13-32). Madrid: Fundamentos.

O'Brien, A. (2018). Women in community radio: a framework of gendered participation. Feminist Media Studies, 19(6), 787-802. DOI: https://doi.org/10.1080/14680777.2 018.1508051

Rodríguez Gómez, E. (2014). El Tribunal Constitucional y el conflicto entre la libertad de información y los derechos al honor, la intimidad y la propia imagen: revisión jurisprudencial. Estudios sobre el Mensaje Periodístico, 20(2), 1209-1224.

Saperas, E. (1985). Los efectos cognitivos de la comunicación de masas. Barcelona: Ariel.

Scheafer, T. (2001). Charismatic Skill and Media Legitimacy. An Actor-Centered Approach to Understanding the Political Communication Competition. Communication Research, 28(6), 711-736.

Scheufele, D. A. (2000). Agenda-setting, priming, and framing revisited: Another look at cognitive effects of political communication. Mass Communication \& Society, 3(2-3), 297-316

Siebert, F. S., Peterson, T. \& Schramm, W. (1956). Four Theories of the Press. Chicago: University of Illinois Press.

Steiner, L. (2005). The feminist cable collective as public sphere activity. Journalism, 6(3), 313-334. DOI: https://doi. org/10.1177/1464884905054063 
Thumin, J. (2004). Inventing television culture men, women, and the box. Oxford: Oxford University Press.

Tuchman, G. (1978). Making News: A Study of the Construction of Reality. Nueva York: The Free Press.

UNESCO (1980). Un solo mundo, voces múltiples. Extraído el 06.12.2019 desde https://unesdoc.unesco.org/ark: /48223/pf0000040066_spa

UNESCO (1983). Principios Internacionales de Ética Profesional del Periodismo. Extraído el 06.12.2019 desde http:// www.editorialdigitaltecdemonterrey.com/materialadicional/p002/capl/el_unesco.pdf

Vallejo, C. (2005). Representación de la violencia contra las mujeres en la prensa española (El País/El Mundo) desde una perspectiva crítica de género. Un análisis crítico del discurso androcéntrico de los medios. Tesis de doctorado no publicada,
Universitat Pompeu Fabra, Facultad de Comunicación, Departamento de Comunicación, Barcelona.

Waterhouse-Watson, D. (2016). News media on trial: towards a feminist ethics of reporting footballer sexual assault trials. Feminist Media Studies, 16(6), 952-967. DOI: https://doi.org/10.1080/14680777.2016.1162827

Yrache Jiménez, L. (2007). Imagen de la mujer y el hombre en publicidad. En Plaza, J. y Delgado C. (Eds.). Género y Comunicación (pp. 101-128). Madrid: Fundamentos.

Zurbano-Berenguer, B. \& García-Gordillo, M. (2017). Methodological proposal for the evaluation of the ethical quality of the news about violence against women. Communication \& Society, 30(1), 73-85. DOI: https://10.15581 /003.30.1.73-85 